\title{
The relationship between binocular brightness matches and luminance difference discriminability of successively presented light flashes'
}

\author{
SIDNEY STECHER ${ }^{2}$ AND MICHAEL SANDBERG \\ BRANDEIS UNIVERSITY
}

\begin{abstract}
Measurements of monocular $\triangle \mathrm{I}$ and PSE as a function of the ISI between two 2-deg foveal fields successively presented to the same retinal area were obtained for two standard durations, using the method of constant stimuli. Binocular brightness matches of the stimuli revealed that detection of a difference occurred whenever a constant difference (in $\log m L$ ) in matching luminance existed. The implication of the results was that $\Delta I$ is related to the rate of change of brightness with changes in test-field luminance.
\end{abstract}

A number of studies have recently been concerned with the relationship between measures of the brightness of a test field and the ability to detect luminance differences (Heinemann, 1961; Novak \& Sperling, 1963; Cornsweet \& Teller, 1965; Nachmias \& Steinman, 1965;Burkhardt, 1966). Some of these investigators have demonstrated circumstances under which brightness and luminance discrimination do not vary in a predictable way.

Heinemann (1961) has pointed to a number of situations in which the "two measures change in opposite directions as a function of changes in the same independent variable [p. 389]." Burkhardt (1966) has suggested that different mechanisms may mediate the perception of field brightness and the detection of luminance differences.

There is another hypothesis, however, that has been suggested by Heinemann (1961). This hypothesis states that luminance discrimination is related not to any particular level of apparent brightness but to the rate at which brightness changes with changes in luminance. Formally stated, $\Delta I=f(d B / d I)$ where the functional relation, $f$, remains to be determined. Thus, if a fixed brightness change is required for a luminance difference between two fields to be detected, this fixed brightness change may result from a variable-luminance change that is a function of the given conditions of stimulation. Whether a fixed change in brightness is brought about by a fixed change in luminance depends upon the rate at which brightness itself changes with changes in luminance under the given conditions. If the brightness changes more rapidly under one condition than under another, then the luminance difference required to yield this fixed brightness change will be less in the condition where brightness changes more rapidly than in the condition where brightness changes less rapidly with changes in luminance.

The history of the experimental investigation of this hypothesis, as well as the difficulty encountered in testing it adequately, has been well summarized by Nachmias and Steinman (1965).

In an investigation of the relationship between these two variables, Heinemann (1961) used the increment method to obtain the luminance-difference thresholds, whereas the brightness matches were made to a test field without the presence of the increment field. There appears to be considerable evidence, however, that an $O$ 's responses in the measurement of luminance-difference thresholds under different stimulation conditions are not based on the same criteria (Stecher, 1968). In the increment-detection method, for example, there is evidence that discriminatirn is mediated by effects near the edges of the test field rather than by an overall brightness evaluation of the fields (Lamar, Hecht, Shlaer, \& Hendley, 1947; Nachman, 1957; Taylor, 1962).

If the aforementioned hypothesis is to be adequately tested, the situation must be so arranged that the marginal contrast effects due to the luminance gradient existing between the increment and background fields are eliminated for both the luminance-difference threshold measurements and the brightness-matching condition. That is, in order to insure that the rate of growth of brightness with changes in luminance is not altered from one situation to the other, the measurements must be made under comparable conditions.

In a recent investigation of this hypothesis, Nachmias and Steinman (1965) used a successive-stimulus presentation procedure to obtain their brightness and discriminability threshold measures. While the authors found qualitative agreement with the hypothesis, some quantitative discrepancies were noted. They pointed out, however, that this may have been due to the lack of comparability of the Ss' task in the two procedures (brightness matching and a signal-detection discriminability measure).

The present investigation studies the relationship between brightness and luminance discrimination, not as a test of the rate-of-change hypothesis, but by empirical determination of the nature of the relationship existing between these two dependent variables under comparable conditions of measurement. The study also differed from all attempts to evaluate the hypothesis in that the luminance range examined was confined to those luminances around which the luminance-discrimination data were determined by the method of constant stimuli.

\section{Apparatus}

The apparatus consisted of a specially constructed four-channel binocular Maxwellian field-viewing system, the complete details of which are presented elsewhere (Stecher, Sandberg, \& Minsky, 1970). The output of the system was generated by two glow-modulator tubes (Sylvania R1131C) that were optically superposed, each eye receiving the combined flux from each of the tubes. The sources could be controlled in duration, frequency, and interval between onsets of the flashes by a Grass Physiological Stimulator (S-8), pulsed through a triode-connected gating circuit. Luminance was controlled by neutral-density wedges and filters placed in an image plane in each of the four channels. Absolute luminance calibrations were made by photometrically matching the Maxwellian fields presented to the right eye to the adapting field presented to the left eye, the adapting field being at a known luminance. The left-eye adapting fields were measured with a Macbeth illuminometer without the 2-mm artificial pupil in place; the Maxwellian field to adapting field matches were made with the artificial pupil in place.

\section{Procedure}

Successive luminance-discrimination measurements. The S was dark-adapted for $15 \mathrm{~min}$ and then looked at the $.003-\mathrm{mL}$, 12-deg 15-min field for 5 more minutes with the right eye. The right eye was used for making the luminance-difference threhold measurements, thus necessitating the use of . 
only one-half of the optical system in the first experiment. The method of constant stimuli was used (without correction), where the $S$ had to indicate whether the second variable-luminance test flash was brighter or dimmer than the first or standard flash. The standard was always $100 \mathrm{~mL}$. A least-square ogive was fitted to the per cent "brighter-than" judgments. A mean and a standard deviation were then computed. The luminance-difference threshold $(\Delta \mathrm{I})$ was taken as the luminance difference between the $50 \%$ value (PSE) and one standard deviation above the mean (Guilford, 1954).

The principal independent variable studied in the first experiment was the temporal separation between the two successively presented 2-deg flashes. The temporal separations (ISI) were measured from the cessation of Flash 1 to the onset of Flash 2. The ISIs used were 95, 120, 150, 180,250 , and $500 \mathrm{msec}$. Two first-flash durations, 320 and $10 \mathrm{msec}$. were investigated, each being set to a standard luminance of $100 \mathrm{~mL}$, while the second test flash was kept at a constant duration of $10 \mathrm{msec}$ for all conditions. The rise time for the $10-\mathrm{msec}$ pulses was about $10 \mu \mathrm{sec}$. In each of the substudies, the appropriate stimulus parameters were randomized. Values of $\Delta I$ and PSE were based on $35-50$ judgments for each of 5-7 test-flash luminances for each of the six ISIs for each first-flash duration for both Ss. The task of the $S$ was to state whether the $10-\mathrm{msec}$ second flash, at different luminances, was brighter or dimmer than the constant-luminance, constant-duration first flash. Two dim red fixation points, located above and below the stimulus array, controlled eye position. The $S$ presented the stimuli to himself after waiting $30 \mathrm{sec}$ between successive trials.

Binocular-brightness matches. The stimulus configuration presented to the right eye was identical in all respects to that in the first experiment. Those luminances of the right-eye second flash (test) for which a psychometric function was obtained in Experiment 1 were now matched by a constant-duration, variable-luminance 2 -deg field presented to the left eye. The matching field was superimposed on the same adapting luminance as the fields in the right eye, and was presented binocularly, spatially adjacent to the right-eye fields. The $S$ saw two flashes in succession in the right eye and only one flash in the left eye. The task of the $S$ was to match the left-eye constant-duration flash to either the first flash in the right eye set to a constant luminance and constant duration or to the second constant-duration flash in the right eye set to the luminances of the psychometric function found in
Experiment 1 between the flashes in the right eye. The $\mathrm{S}$ controlled the luminance of the matching field by activating a switch that permitted a current to turn a motor that governed the position of a two-log-unit circular neutral-density wedge.

During each daily session, a first-flash duration would be set. For each first- and second-flash combination, an ISI would be randomly selected by the $E$. For this condition, a randomized tape would select one of those luminances of the second flash for which a value of $\Delta I$ had been previously determined. The $\mathrm{S}$ would then expose the display and adjust the flash in the left eye to match the first and second flashes in the right eye. The $S$ was allowed an unlimited number of exposures until he was satisfied that a match was obtained. After making such a match, the $S$ would press the advance key. The tape reader would advance the wedge to another luminance of the second flash for which the luminancediscrimination measurements were previously made. Ten to 15 such matches were made for each of six to nine test-field luminances for each of the six ISIs. The $\mathrm{E}$ recorded the setting of the matching wedge during nonautomated recording trials, and monitored the CRO display of the pulses. Two $S s$ were used, although the results are presented for only one.

\section{Results and Conclusions}

Successive luminance-difference threholds. Figure 1 shows the results obtained for $\triangle I$ and PSE in $\log \mathrm{mL}$ as a function of $\log$ ISI (milliseconds).

The results indicate that sensitivity is poor with short separations between the flashes. With increases in the separation between the flashes, sensitivity increases $(\Delta \mathrm{I}$ decreases). The extent of the decrease in $\Delta I$ is about .6-.7 log unit for both standard conditions ( 320 and $10 \mathrm{msec}$ ). The PSE in the 10 -msec-ISI-10-msec condition is relatively horizontal, whereas the PSEs obtained in the 320 -msec-ISI-10-msec condition are more variable for the various ISIs. In neither case are the changes in PSE as great as that found for $\Delta I$. Increases in first-flash duration lead to increases in $\Delta I$ and to increases in the luminance of the second flash required to obtain the PSE. These results have been confirmed using another $S$. The 10-msec-ISI-10-msec condition is highly similar to that previously found by this author (Stecher, 1967).

Binocular brightness matches of successive flashes. In Fig. 2, a 10-msec flash presented to the left eye was varied by the $S$ to match one or the other of the $10 \mathrm{msec}$ flashes presented to the right eye.

The legend "condition" indicates that the left eye received one flash that was coincident in time and of the same duration

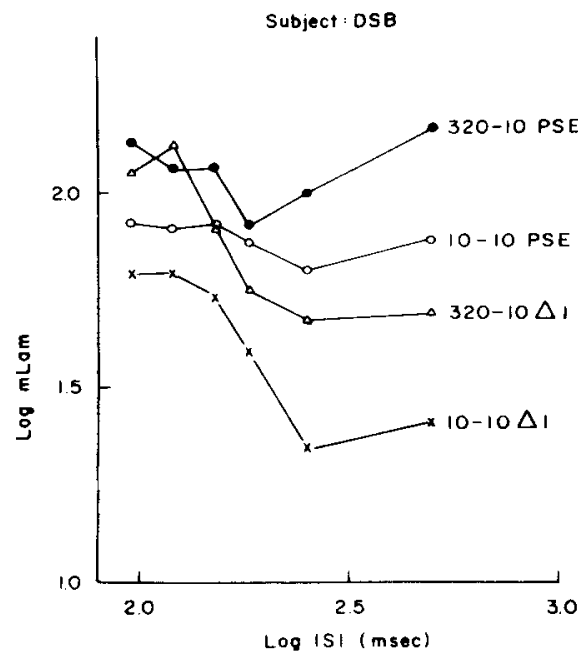

Fig. 1. $\triangle I$ and $P S E$ in $\log \mathrm{mL}$ as a function of $\log$ ISI (milliseconds). Two conditions of first- and second-flash durations are presented: a $320-\mathrm{msec}$ standard flash followed by a 10-msec test flash, and a 10 -msec standard flash followed by a 10-msec test flash. In both cases, the first flash (standard) was set to $100 \mathrm{~mL}$, and the task of the $S$ was to state whether the second flash set to various luminances was "brighter" or "dimmer" then the first. Viewing was monocular, and flashes were presented to the same retinal areas.

as the first of the paired flashes to the right eye. The left-eye flash was matched to the second of the right-eye flashes when the latter were set to those luminances for which the luminance-discrimination judgments were previously made. The brightness matches to the second flash thus depict the comparison matches of the psychometric function values. These functions are shown in Graphs A-F, for each ISI investigated (95, $120,150,180,250$, and $500 \mathrm{msec}$ ). Graph G in Fig. 3 represents the mean matching value to the first right-eye flash set to $100 \mathrm{~mL}$ in the presence of the second flash set to the luminances indicated on the abscissa taken across ISI. The first-flash matching values were averaged across ISI because the first-flash matches were found to be independent of the interval between the successive flashes presented to the right eye. A straight line was fitted to the data in log-log coordinates, using a least-square criterion. The residual variance was negligible.

Inspection of the various functions reveals that the slopes of the matching functions to the second flash change as the ISI changes. Matches to the first flash, however, indicate that changes in second-flash luminance exert a negligible effect on the brightness of Flash 1 (retroactive masking) over the range of ISI 

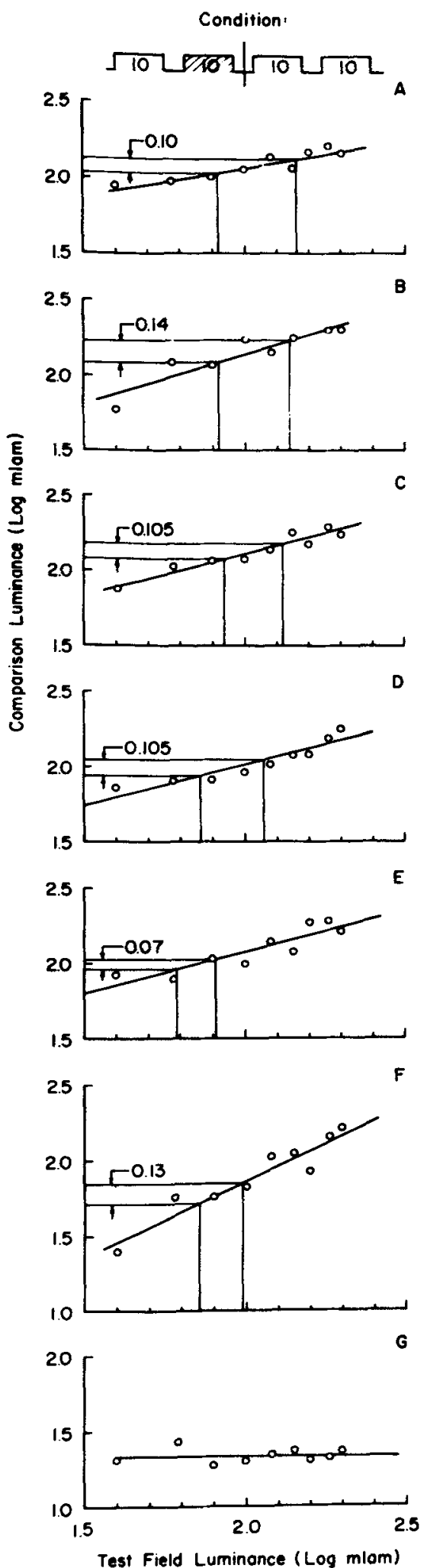

Fig. 2. Brightness-matching functions. The legend, "condition," refers to the displays presented to each eye. The right eye received two $10-\mathrm{msec}$ flashes in succession, the first flash being set to $100 \mathrm{~mL}$, while the second was set to the luminances of the psychometric function as indicated on the abscissa. The left eye received a 10 -msec flash coincident in onset and of the same duration as the first right-eye flash, the left-eye flash being varied in luminance to match the right-eye flashes. These values are represented on the ordinate, in $\log \mathrm{mL}$. Graphs A-F refer to the matching results obtained for various ISls between the right-eye flashes $(95,120,150,180,250$, and 500 msec, respectively). Graph $G$ refers to the mean matches to the first right-eye flash. A least-square line was fit to the data in log-log coordinates. The two vertical lines along the abscissa refer to the PSE and $\Delta I$ obtained for these conditions in Experiment 1. The horizontal lines to the ordinate represent the matching luminance (in $\log \mathrm{mL}$ ) required to yield the obtained difference between PSE and $\Delta I$.

investigated. Graphs A-F indicate that as the test-flash luminance is increased, the comparison luminance also increases. As can be seen from the slopes, this increase in comparison luminance (in $\log \mathrm{mL}$ ) changes more rapidly at longer than at shorter ISIs.

Figure 3 presents the condition where the right eye was presented with a $320-\mathrm{msec}$ flash set to $100 \mathrm{~mL}$, and was succeeded after various ISIs by a $10-\mathrm{msec}$ flash set to the various luminances for which a psychometric function was previously obtained and which are indicated on the abscissa.

The left eye received one flash of 320-msec duration that was coincident in onset with the first flash presented to the right eye. The luminance of the left-eye flash was adjusted by the $S$ to match one or the other of the two righteye flashes, the first being set to the standard luminance of $100 \mathrm{~mL}$ and the second to the luminances of the previously determined psychometric - functions as indicated on the abscissa. Graphs A-F refer to the obtained matches to the second flash for the various ISIs that were used in previous conditions. A straight line has been fit to the data in log-log coordinates, using a least-square criterion. These results also indicate that as the luminance of the test flash is increased, the comparison luminance required for a match also increases, this increase in comparison luminance changing more rapidly at longer than at shorter ISIs.

The slopes of the second-flash matching functions indicate the rate at which the comparison luminance (in $\log \mathrm{mL}$ ) changed with respect to changes in the test-flash luminance (in $\log \mathrm{mL}$ ). Thus, $\Delta \log B / \Delta \log \mathrm{L}$ yields directly the rate of change under the various conditions of measurement. Inspection of Figs. 2 and 3 reveals that the

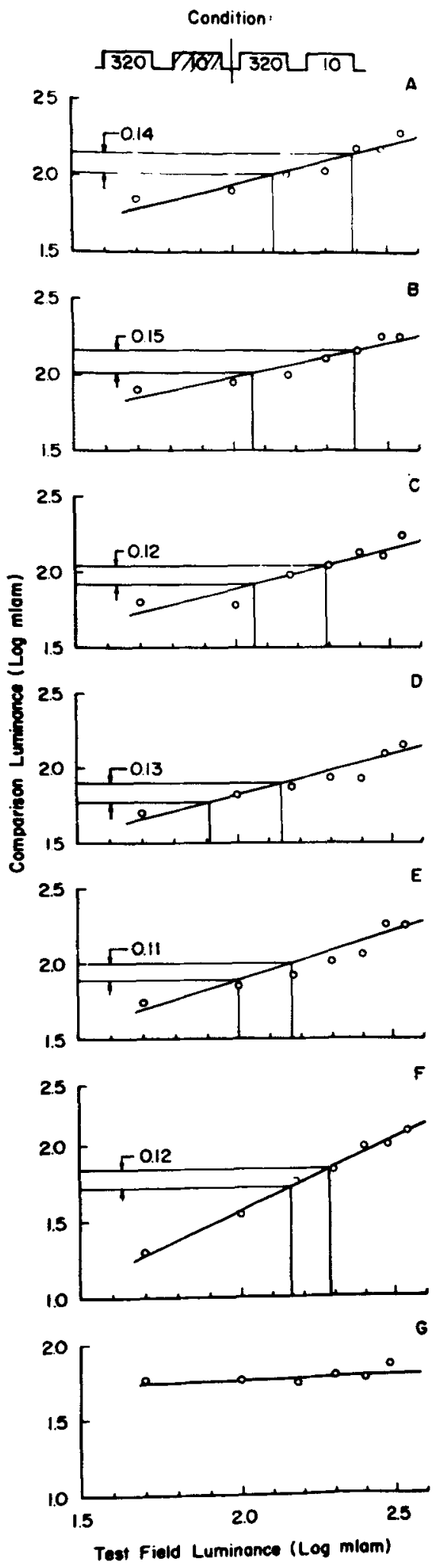

Fig. 3. Brightness-matching functions. The legend, "condition," refers to the displays presented to each eye. The right eye received a $100-\mathrm{mL}, 320-\mathrm{msec}$ flash followed, 
$95,120,150,180,250$, or 500 msec after its cessation, by a $10-\mathrm{msec}$ flash set to the luminances indicated on the abscissa and for which a psychometric function had previously been obtained. The left eye received a $320-m s e c$ flash that was as the right-eye flashes. The ordinate represents the comparison luminance (in $\log \mathrm{mL}$ ) found to match the right-eye flashes. Graphs A-F refer to the various ISIs. Graph $G$ represents the matches to the first flash in the presence of the second flash set to the abscissa luminances averaged across ISI. The vertical lines along the abscissa represent the PSEand $\Delta I$ values found in the luminance-discrimination study. The two horizontal lines along the ordinate represent the comparison luminance difference (in $\log \mathrm{mL}$ ) required to yield $\Delta I$. coincident in time and of the same duration

ISI influences the rate at which comparison luminance changes with respect to changes in second-flash luminance.

Figure 4 is a plot of the slopes obtained at all ISIs for both standard-flash duration conditions.

The results indicate that there are no important differences in the shapes of the functions obtained under each condition of stimulation. At long ISIs, a unit increase in second-flash luminance yields a unit increase in matching luminance (slope $\cong 1.0$ ). At short ISIs, a unit change in second-flash luminance yields much less than a unit change in matching luminance (slope $\cong 0.5$ ). Thus, the rate of growth in matching luminance is less at shorter ISIs than at longer ISIs. The slope analysis reveals that as the ISI is decreased, the rate of change of matching luminance is depressed.

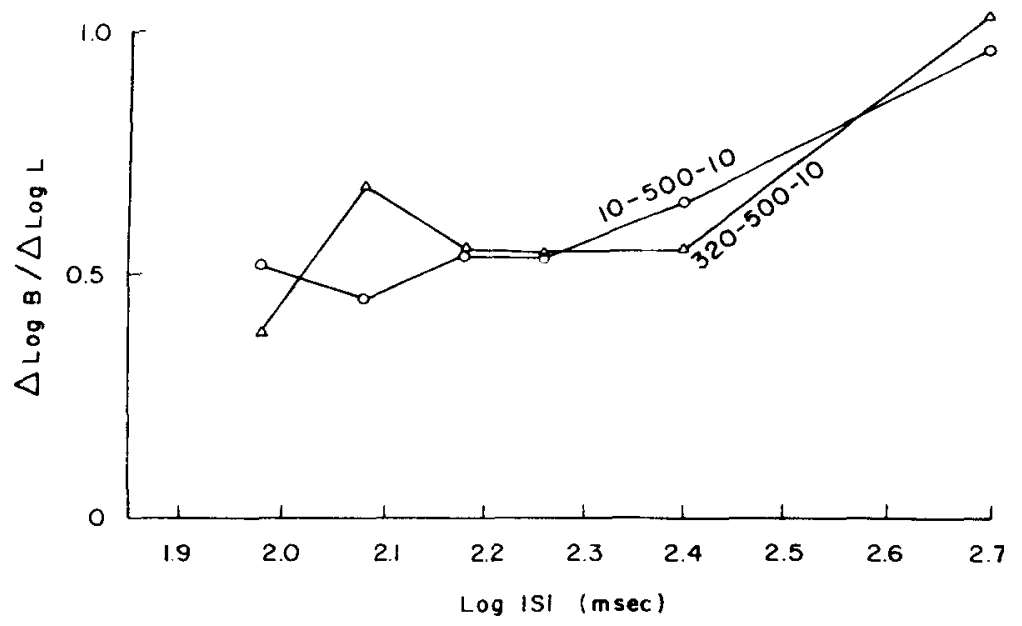

Fig. 4. Slopes of the matching functions as a function of $\log$ ISI (milliseconds) for both conditions of standard-flash duration ( 320 and $10 \mathrm{msec}$ ).

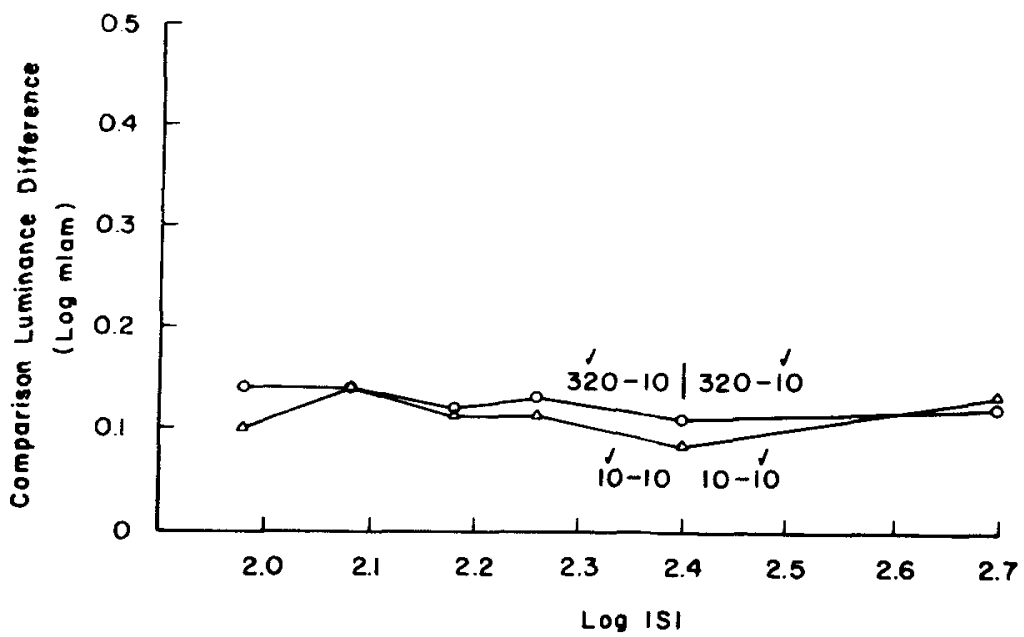

Fig. 5. Matching-luminance differences (in $\log \mathrm{mL}$ ) as a function of $\log$ ISI (milliseconds) for both conditions of measurement. These values were obtained from Figs. 3 and 4.
Evidence from previous studies (Stecher, 1968) indicates that relative discriminability is also hampered by increasing temporal interaction between successively presented fields.

In order to evaluate the relationship between the brightness of the flashes and successive luminance detectability, it must be recalled that $\Delta I$ was obtained as that stimulus difference between the mean (PSE) and one standard deviation above the mean of the psychometric function. The PSE, when obtained by the present procedure, is that luminance of the test stimulus required monocularly to match the standard. It is around this value that $\Delta I$ is determined (Nachmias \& Steinman, 1965; McDougall, 1904; Stecher, 1968). In the case where $\mathrm{PSE}=\mathrm{POE}, \triangle \mathrm{I}$ is determined around the objective value of the standard flash. Where $P S E \neq P O E, \Delta I$ is determined about a value of the test flash that monocularly matches the standard. If the relationship between $\Delta I$ and the brightness matches is to be correctly determined, these facts must be considered. It is possible, in the present case, for the luminance of PSE $+\Delta \mathrm{I}$ to be $<\mathrm{I}$, where $\mathrm{I}$ is the objective value of the standard.

In each of the graphs A-F in Figs. 2 and 3, two values have been marked off along the abscissa. These values correspond to the luminances of the PSE and $\Delta \mathrm{I}$ obtained for each condition in the successive luminance-discrimination study. The differences between these PSE and $\Delta I$ values in $\log \mathrm{mL}$ are the values plotted in Fig. 1 for the respective conditions. To each of these values of PSE and $\Delta \mathrm{I}$, there corresponds, on the ordinate of Figs. 2 and 3 , a matching luminance value. The difference between these matching luminances (in $\log \mathrm{mL}$ ) is the matching-luminance difference (in $\log \mathrm{mL}$ ) required to yield a difference of one standard deviation along the psychometric function. These matching-luminance differences will depend upon the size of $\Delta I$ and the slope of the matching functions. For a constant luminance-difference threshold, if the slope of the matching function is steep (e.g., if the rate of change of matching luminance with changes in test-field luminance is great), a large matching-luminance difference will be required to yield the constant detectability; if the slope of the matching function is relatively flat (if brightness grows slowly with changes in luminance), then a much smaller matching-luminance difference will be required to yield the constant-luminance difference. If, however, a constant difference in matching luminance is required in order for a difference to be detected, then a steep matching-luminance function would yield a small luminance threshold, while a flat slope would require a greater value of $\Delta I$ in order to obtain the same matching-luminance difference. 
In the present investigation, the values of $\Delta \mathrm{I}$ were found to change over a range of .6-.7 $\log$ units, while the slopes of the matching functions ranged from 0.5 at short ISIs to 1.0 at long ISIs. Figure 5 shows the matching-luminance differences (in $\log \mathrm{mL}$ ) obtained for all ISIs and both conditions of testing.

The results indicate that a constant difference in matching luminance (in $\log \mathrm{mL}$ ) is required for a constant detectability for all ISIs and for all conditions of stimulation, despite the .6-.7 log unit change in $\Delta I$. As the temporal interaction between the successively presented flashes is increased, i.e., as ISI is decreased, the slope of the matching function decreases. In order to obtain a constant difference in matching luminance, the difference between PSE and $\Delta \mathrm{l}$ must be increased. With a decrease in temporal interaction between the flashes, i.e., as ISI is increased, the brightness of the test flash changes more rapidly with changes in test-field luminance, and a smaller value of $\Delta \mathrm{I}$ is required in order to yield the same criterion difference in comparison luminance.

These results indicate that it is not necessary to postulate two different mechanisms of operation for field brightness and luminance discriminability (Burkhardt, 1966). Rather, the results imply that luminance discrimination is related to the rate of change of brightness of the test flash with changes in test-flash luminance (see Heinemann, 1961). Further, this rate of change is influenced by the temporal interaction between the successively presented fields. Present investigations are exploring this analysis over a greater range of luminances and durations and for conditions of simultaneously presented, spatially adjacent stimulations. Studies are also being planned in the tactile modality using a similar method of analysis.

\section{REFERENCES}

BURKHARDT, D. Brightness and the increment threshold. Journal of the Optical Society of America, 1966, 56, 979.

CORNSWEET, T. M., \& TELLER, D. Y. Relation of increment thresholds to brightness and luminance. Journal of the Optical Society of America, 1965, 55, 1303.

GUILFORD, J. P. Psychometric methods. New York: McGraw-Hill, 1954.

HEINEMANN, E. G. The relation of apparent brightness to the threshold for differences in luminance. Journal of Experimental Psychology, 1961, 61, 389-399.

LAMAR, E. S., HECHT, S., SHLAER, S., \& HENDLEY, C. D. Size, shape and contrast in detection of targets by daylight vision: 1 . Journal of the Optical Society of America. 1947, 37, 531-545.

McDOUGALL, W. The variation of the intensity of visual sensation with the duration of the stimulus. British Journal of Psychology, 1904, 1, 151-189.

NACHMAN, M. The influence of size and shape on the discrimination of visual intensity. American
Journal of Psychology, 1957, 70, 211-218.

NACHMIAS, J., \& STEINMAN, R. M. Brightness and discriminability of light flashes. Vision Research, 1965, 5, 545-557.

NOVAK, S., \& SPERLING, G. Visual thresholds near a continuously visible or a briefly presented light-dark boundary. Optica Acta $1963,10,1-5$.

STECHER, S. Discrimination of luminance differences between temporally separated paired flashes. Joumal of the Optical Society of America, 1967, 57, 1271-1272.

STECHER, $S$. Temporal and spatial effects in luminance discrimination. Perception \& Psychophysics, 1968. 4. 155-158.

STECHER, S., SANDBERG, B., \& MINSKY, P. J. Successive luminance difference thresholds and brightness as a function of the interstimulus interval and durations of successive flashes. Perception \& Psychophysics, 1970, 7, 79-85.

TAYLOR, N. W. Foveal vision: Dependence of threshoid energy on the visual angle of a circular target. Joumal of the Optical Society of America, 1962, 52, 820-825.

\section{NOTES}

1. This research was supported by Grant R01-NB-07340-01 from the National Institute of Neurological Diseases and Blindness, United States Public Health Service, to the senior author. The authors wish to thank Drs. Eric Heinemann and David Raab for discussion of the manu script.

2. Address: Department of Psychology, Brandeis University, Waltham, Massachusetts 02154.

(Accepted for publication May 13, 1969.) 